\title{
Design of Temperature Acquisition System Based on STC89C52RC Single-Chip Microcomputer
}

\author{
Yu-Ping Su, Ning Cai \\ College of Electrical Engineering, \\ Northwest University for Nationalities, \\ Lanzhou, China 730124 \\ E-mail: syp_abc@163.com,caining91@tsinghua.org.cn
}

\begin{abstract}
The temperature is an important physical quantity that is closely related to daily production and life. We need extract temperature information on many occasions. Temperature acquisition system based on signal chip machine, temperature sensor, serial communication and VB knowledge is designed in the paper. The system can read temperature data from the sensor, and then display it on the liquid crystal screen. At the same time temperature value is transmitted to the PC through the serial communication, PC can display the real-time temperature value. The test results show that the system has advantages of rapid response, and can better display the actual environment temperature, moreover, the system circuit design is simple with lower cost and smaller size, and can be applied in many fields of temperature detection.
\end{abstract}

Keywords-temperature acquisition; liquid crystal screen; serial communication; $p c$

\section{INTRODUCTION}

The temperature is a basic environment parameter, so monitoring temperature is important in industrial production and life. The traditional method of temperature measurement adopts thermocouple or thermistor which gets temperature though analog-digital conversion with quite complicated hardware structure [1][2]. Integrated temperature sensor has the advantages of good linearity, high stability, strong interchangeability, being easy to process and has been widely used in many places. In recent years, DS18B20 as a representative of the new digital temperature sensor plays an important role in temperature detection. Temperature acquisition system based on SCM and DS18B20 is introduced in this paper. The system builds the connection between SCM and PC by using serial communication, monitoring interface is designed through the VB6.0 software. The system can conveniently realize the functions of temperature acquisition and temperature display.

\section{SYSTEM SCHEMATIC DIAGRAM}

The temperature acquisition system is composed of an SCM chip (STC89C52RC), a temperature sensor (DS18B20), a serial communication module (RS232), an LCD module (LCD1602) and a PC. The system schematic diagram is shown in figure 1 .

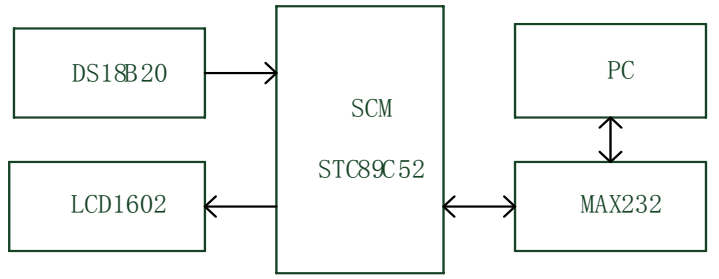

Figure 1. System schematic diagram

The data acquisition process is as follows: the STC89C52RC gets temperature value from the temperature sensor, then transmits it to LCD circuit after information processing, and uploads to computer through serial communication, finally the computer displays the temperature value in real- time.

\section{SYSTEM HARDWARE DESIGN}

\section{A. Main Control Circuit and Reset Circuit}

The reason why system uses the STC89C52 microcontroller is that STC89C52 has in-system programming (ISP) characteristic; store chip can be programmed without removing from embedded device [3]. It can reduce the cost, and improve the performance. An STC89C52, a crystal oscillator circuit, and a reset circuit constitute a minimum system.

\section{B. Temperature Collection Circuit}

It is essential to guarantee the power supply for the I/0 lines to enable DS18B20 to make accurate temperature conversion during the conversion process. With considering the fact that system is only for a single point measurement, we connect an external power from the VDD pin. Port P3.2 is connected to the DQ terminal of DS18B20, the temperature information will be transformed to SCM. The temperature collection circuit is illustrated in figure 2 . 


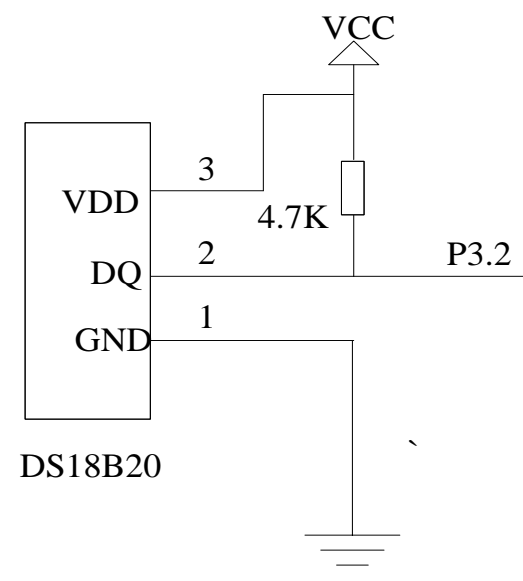

Figure 2. Temperature collection circuit.

\section{Level Conversion Circuit}

The RS232 logic level is not compatible with TTL level, and serial port of SCM is not directly connected to RS232 interface of PC. Therefore, an external electrical level conversion circuit is required in order to realize the connection. The system adopts MAX232 chip. The interface circuit is shown in figure 3, P3.0 port and P3.1 port of SCM are linked to T1IN, R1OUT of MAX232 to realize the serial communication between the two [4][5].

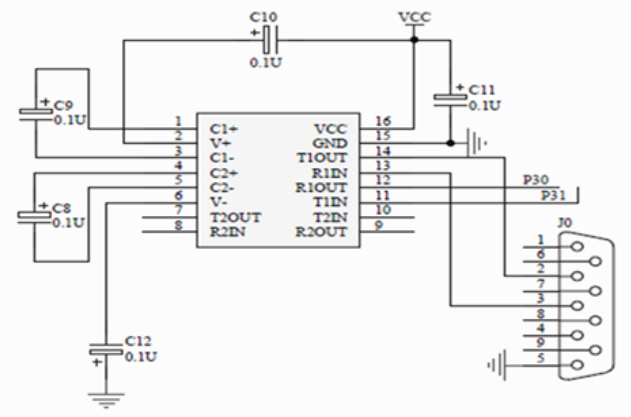

Figure 3. Interface circuit.

\section{Display Circuit}

LCD1602 is a common liquid crystal display screen. It is easy to read and write and has high accuracy. It takes up less resources, can display stable data, and has strong antiinterference ability compared to dynamic digital tube. Display circuit is shown in figure 4. In the graph, DB0 DB7 of LCD 1602 are 8 data terminals, which are connected to P1.0 P1.7 port of SCM. E terminal, RW terminal, RS terminal are control terminals, which are connected to P2.5 port, P2.6 port and P2.7 port of SCM respectively. LED-, LED+ are connected with adjustable resistor, constituting the back-light control [6]. The adjustable resistor R1 is used for adjusting the liquid crystal contrast.

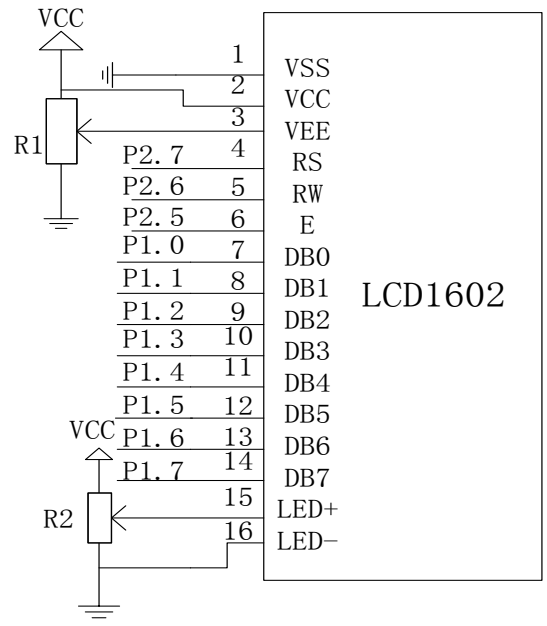

Figure 4. Display circuit.

\section{SYSTEM SOFTWARE DESIGN}

The software of temperature acquisition system is mainly developed by $\mathrm{C}$ language. In order to facilitate debugging and maintenance, we use modularization programming ideas. The software includes the main program, the DS18B20 temperature sensor module program and the LCD1602 display module program [7][8].

The work procedure of the main program is as follows: First we make the system initiation and setup the DS18B20 and the LCD1602; then system reads the temperature value and converts it and transmits the converted value to the LCD. The SCM monitors signal of upper computer constantly, judging whether upper computer send request data or not. Once the correct request is detected, the system will enter the interrupt service program, and the SCM will send temperature data to computer. The flow chart of main program is shown in figure 5 .

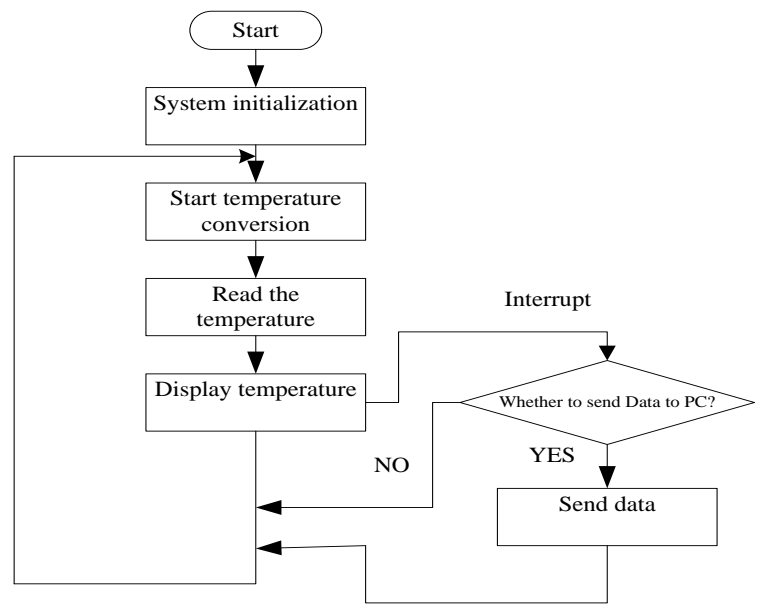

Figure 5. Flow chart of main program.

Data acquisition system regards the STC89C52 chip as core; STC89C52 controls reset, read and write of the DS18B20. DS18B20 is the latest single-wire digital 
temperature sensor. System can directly get digital signal of temperature value from it.With its high accuracy and precision, high resolution and good anti-interference, data need not be checked. Due to data format read is binary, system must convert them from binary to decimal in displaying the temperature value. The temperature acquisition process is shown in figure 6.

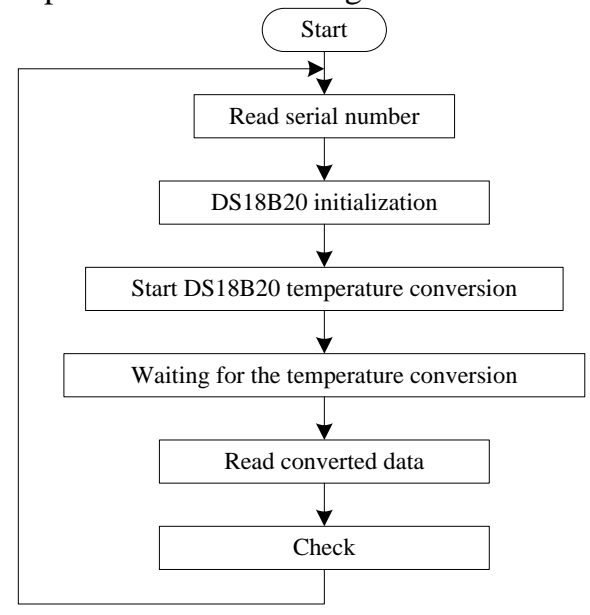

Figure 6. The temperature acquisition process.

LCD1602 is a display module. System needs to read its state before sending data or commands every time. System can send valid data or commands only if LCD1602 is not busy. Otherwise errors will occur. The program flow diagram is shown in figure 7.

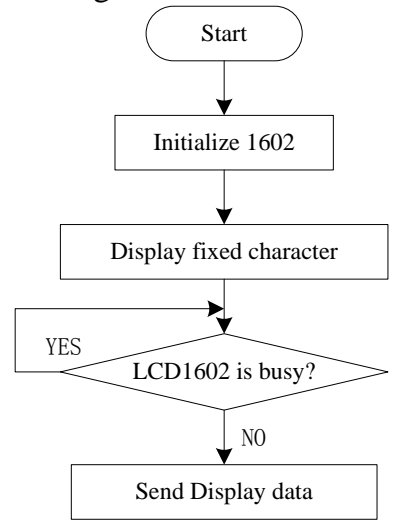

Figure7. The flow diagram of display program.

\section{RS-232 SERIAL COMMUNICATION PROGRAM}

The collected temperature data can be sent to the computer for the convenience of remote control and monitor real-time temperature value. System can send data to the terminal device through RS-232. The program flow diagram is shown in figure 8.

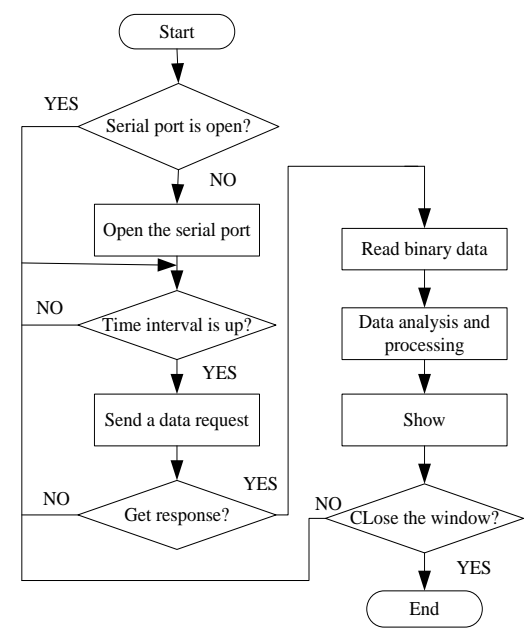

Figure 8. The flow chart of serial communication program.

VB6.0 can develop a friendly user interface quickly and easily. The monitoring software of temperature acquisition system is completed by the VB6.0. There are two main methods in development of serial communication program. One is the use of MSComm serial control; the other is calling window API functions. The former is quicker and more convenient than the method which calls the API dynamic link library. Moreover, it can realize same function by less code, greatly improving the programming efficiency and stability of system. The PC and the SCM communication can be implemented by using MSComm communication control [9]. The interface of the monitoring software is shown in figure 9, from which the real-time temperature data can be seen.

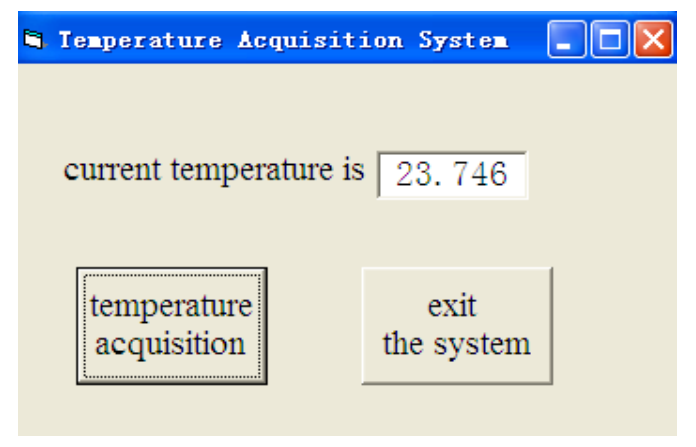

Figure 9. The interface of the monitoring software.

\section{CONCLUSION}

A temperature acquisition system is designed in the article. It can accomplish data acquisition, data processing, data display and data transmission in real-time, and can be widely used in agricultural production, scientific research and other fields, such as grain depot, green house, fresh storage, etc. It has broad application prospect. The system uses the DS18B20 digital sensor, which includes the temperature sensor and A/D conversion and help to greatly improve the reliability of the system, making the entire circuit simple. The length of signal cable does not affect the 
acquisition accuracy. Due to its low cost and wide application range, it can save a lot of manpower, material and financial resources, and bring considerable economic benefits, which are evidently advantageous over than the conventional artificial monitoring. Therefore, we can select proper sensors and control equipment for specific facilities in order to make the system more generally applicable. In addition, the system software is flexible. If only it is slightly modified, the application system can be rapidly reassembled.

\section{ACKNOWLEDGMENT}

This work was supported by National Natural Science Foundation of China (Grants 61263002 \& 61174067), and by the Fundamental Research Funds for the Central Universities, Northwest University for Nationalities (Grant 31920150013).

\section{REFERENCES}

[1] Daqing Shi, "Design of a digital thermometer", Experiment Science and Technology,vol.10, no.1, pp. 178-180, 2012. (in Chinese)
[2] Linzhi Wang, Yuxian Li, "The design of digital thermometer based on DS18B20", Industrial Instrumentation and Automation, no.5, pp.74-76, 2011. (in Chinese)

[3] Tianxiang Guo, New Concept 51 Single-Chip Microcomputer C Language Tutorial, Publishing House of Electronics Industry, 2009. (in Chinese)

[4] Yizhi Fan, Liyuan Chen, Visual Basic and RS-232 Serial Communication Control, Tsinghua University Press, 2002. (in Chinese)

[5] Fan Yang, Hua Wang, "Temperature measurement and control system based on AT89S52", Electrical Automation, vol.3, no.3, pp.53-55, 2009. (in Chinese)

[6] Jianchun Zhou, "Temperature measurement system based on the micro controller and the PC serial communication”, Master's Thesis (Su Zhou University), 2011. (in Chinese)

[7] Wei Peng, Single-Chip Microcomputer C Language Program Design Training of 100 Cases Based on 8051+Proteus Simulation, Electronic Industry Press, 2009. (in Chinese)

[8] Dongfeng Wang, Guanqiang Dong, Single-Chip Microcomputer C Language 100 Examples of Application, Electronic Industry Press, 2009. (in Chinese)

[9] Yanjie Qi, Hao Wu, Wenchao Song, Visual Basic Serial Communication Engineering Development Instance of Navigation, People's Posts and Telecommunications Press, 2003. (in Chinese) 